\title{
RADIAL GROWTH AND VARIATION OF BOUNDED ANALYTIC FUNCTIONS
}

\author{
by D. J. HALLENBECK and T. H. MACGREGOR
}

(Received 17th March 1987)

\section{Introduction}

If a function $f$ analytic in $\Delta=\{z \in \mathbb{C}:|z|<1\}$ has a nontangential limit as $z \rightarrow e^{i \theta}$, then $\lim _{r \rightarrow 1^{-}}(1-r) f^{\prime}\left(r e^{i \theta}\right)=0[7$, p. 181). It follows that this limit is zero for almost all $\theta$ for a number of classes of functions including the set $H^{\infty}$ of bounded analytic functions. In this paper we prove that this result for $H^{\infty}$ is sharp in a strong sense.

If $f$ is also univalent in $\Delta$, then the growth of $f^{\prime}$ is much more restricted. In particular, such a function satisfies $\lim _{r \rightarrow 1}(1-r)^{\alpha} f^{\prime}\left(r e^{i \theta}\right)=0$ for almost all $\theta$ for each $\alpha>0$ [1]. The sharp result of this kind was proved by N. G. Makarov in [5].

We consider the same problem for a function subordinate to a univalent function. If $f$ and $F$ are analytic functions, then $f$ is called subordinate to $F$ provided that there is an analytic function $\phi$ so that $\phi(0)=0,|\phi(z)|<1$ for $|z|<1$ and $f=F \cdot \phi$. We show that if $f$ is subordinate to a univalent function $F$, then $\lim _{r \rightarrow 1}-(1-r)^{p} f^{(p)}\left(r e^{i \theta}\right)=0$ for almost all $\theta(p=1,2, \ldots)$. We also show that this result is sharp. When $p=1$ this is strikingly different from the sharp result for univalent $F$ in [5].

In the last part of this paper, we construct examples to show that certain estimates on the radial growth and variation of bounded analytic functions and their higher derivatives are sharp. If $f$ is analytic in $\Delta$ and satisfies $|f(z)| \leqq M$ for $|z|<1$, then $[3$, p. 330]

$$
\left|f^{\prime}(z)\right| \leqq \frac{M}{1-|z|^{2}}
$$

for $|z|<1$. Equality holds in (1) at $z=z_{0}$ only for the functions

$$
f(z)=A \frac{z-z_{0}}{1-\bar{z}_{0} z}
$$

where $|A|=M$.

Let $M(r, f)=\max _{|z|=r}|f(z)|$ whenever $f$ is analytic in $\{z:|z| \leqq r\}$. If $f \in H^{\infty}$ then (1) holds and this implies

$$
M\left(r, f^{(p)}\right)=O\left[\frac{1}{(1-r)^{p}}\right]
$$


for $p=1,2, \ldots$ and $0<r<1$. It follows directly from (3) that

$$
\int_{0}^{r}\left|f^{(p)}\left(x e^{i \theta}\right)\right| d x= \begin{cases}0\left[\log \frac{1}{1-r}\right], & \text { if } \quad p=1 \\ 0\left[\frac{1}{(1-r)^{p-1}}\right], & \text { if } \quad p>1 .\end{cases}
$$

Since equality in (1) occurs only for different functions for different values of $z_{0}$ it is not clear whether there is a bounded analytic function having the growth exhibited by the right hand sides of (3) or (4) as $r \rightarrow 1$.

In [4] it was shown that there is an $f \in H^{\infty}$ for which $\varlimsup_{r \rightarrow 1}(1-r) M\left(r, f^{\prime}\right)>0$. We give two examples which are quite different from each other and verify that the growth in (3) and (4) can be attained. In fact there is an $f \in H^{\infty}$ for which $\underline{\lim }_{r \rightarrow 1-}(1-r)^{p} M\left(r, f^{(p)}\right)>0$ for $p=1,2, \ldots$.

\section{Radial growth}

The argument given in [7, p. 181-182] can be applied to the Cauchy formula for $f^{(p)}$ to show that if $f$ has a nontangential limit as $z \rightarrow e^{i \theta}$, then $\lim _{r \rightarrow 1}-(1-r)^{p} f^{(p)}\left(r e^{i \theta}\right)=0$ for $p=1,2, \ldots$. This implies that if $f \in H^{\infty}$ then $\lim _{r \rightarrow 1^{-}}(1-r)^{p} f^{(p)}\left(r e^{i \theta}\right)=0$ for almost all $\theta$ and for every $p$. We next prove that this result is sharp in the following sense.

Theorem 1. Let $\varepsilon$ be a positive nonincreasing function on $(0,1)$ such that $\lim _{r \rightarrow 1}-\varepsilon(r)=0$. Then there is a function $f$ that is bounded and analytic in $\Delta$ for which

$$
\varlimsup_{r \rightarrow 1^{-}}\left\{\frac{(1-r)^{p} \min _{|z|=r}\left|f^{(p)}(z)\right|}{\varepsilon(r)}\right\}=+\infty
$$

for $p=1,2, \ldots$

Proof. It suffices to show that such a function $f$ exists which has the following property: there is a sequence $\left\{r_{k}\right\}$ such that $0 \leqq r_{k}<1, \lim _{k \rightarrow \infty} r_{k}=1$ and for every $\theta$ and every $p$,

$$
\left(1-r_{k}\right)^{p}\left|f^{(p)}\left(r_{k} e^{i \theta}\right)\right| \geqq \frac{\varepsilon\left(r_{k}\right)}{6}
$$

for all sufficiently large values of $k$. It is clear that (6) implies (5) by first applying (6) with $\varepsilon$ replaced by $\sqrt{\varepsilon}$.

Let

$$
f(z)=\sum_{n=1}^{\infty} \frac{1}{n^{2}} z^{\lambda_{n}}
$$


where $\left\{\lambda_{n}\right\}$ is a strictly increasing sequence of positive integers, suitably selected in terms of $\varepsilon$ in a manner described below. For any choice of $\left\{\lambda_{n}\right\}$ it follows that

$$
|f(z)| \leqq \sum_{n=1}^{\infty} \frac{1}{n^{2}} \text { for }|z| \leqq 1
$$

and hence $f$ is a bounded analytic function.

The sequence $\left\{\lambda_{n}\right\}$ can be defined so that the following three inequalities hold for all positive integers $k$ :

$$
\begin{gathered}
\lambda_{k+1} \geqq \lambda_{k}^{2} ; \\
\varepsilon\left(1-\frac{1}{\lambda_{k}}\right) \leqq \frac{1}{k^{2}} ; \\
\lambda_{k+1}\left(\lambda_{k+1}-1\right) \geqq \frac{(k+1)^{3}}{n^{2}} \lambda_{n}\left(\lambda_{n}-1\right)
\end{gathered}
$$

for $n=1,2, \ldots, k$. As we show, each of (8), (9) and (10) are conditions which can be inductively satisfied so that at any stage in the induction the next term in the sequence $\left\{\lambda_{n}\right\}$ is permitted to take on all arbitrarily large values; thus there is a sequence satisfying all three conditions. This claim is clear for (8). The same is the case for (9) because $\varepsilon$ is nonincreasing and $\lim _{r \rightarrow 1}-\varepsilon(r)=0$. The numbers on the right hand side of (10) form a finite set and thus (10) holds for all large values of $\lambda_{k+1}$, given $\lambda_{1}, \lambda_{2}, \ldots, \lambda_{k}$.

Let $r_{n}=1-\left(1 / \lambda_{n}\right)$ for $n=1,2, \ldots$, and let $k$ be any positive integer. Then

$$
f^{(p)}\left(r_{k} e^{i \theta}\right)=A+B+C
$$

where

$$
\begin{gathered}
A=\sum_{n=1}^{k-1} \frac{\lambda_{n}\left(\lambda_{n}-1\right) \ldots\left(\lambda_{n}-(p-1)\right)}{n^{2}}\left(r_{k} e^{i \theta)^{\lambda_{n}-p},}\right. \\
B=\frac{\lambda_{k}\left(\lambda_{k}-1\right) \ldots\left(\lambda_{k}-(p-1)\right)}{k^{2}}\left(r_{k} e^{i \theta)^{\lambda_{k}-p}}\right.
\end{gathered}
$$

and

$$
C=\sum_{n=k+1}^{\infty} \frac{\lambda_{n}\left(\lambda_{n}-1\right) \ldots\left(\lambda_{n}-(p-1)\right)}{n^{2}}\left(r_{k} e^{i \vartheta}\right)^{\lambda_{n}-p}
$$

We are only interested in $k$ large and so assume that $\lambda_{k}>p$. Also the sum in (12) takes place for values of $n$ for which $\lambda_{n} \geqq p$. 
Inequality (10) and the fact that $\left\{\lambda_{n}\right\}$ is increasing imply that

$$
\begin{aligned}
|A| \leqq & 2^{p}\left\{\sum_{n=1}^{k-2} \frac{\lambda_{n}\left(\lambda_{n}-1\right) \ldots\left(\lambda_{n}-(p-1)\right)}{n^{2}}\left(1-\frac{1}{\lambda_{k}}\right)^{\lambda_{n}}\right. \\
& \left.+\frac{\lambda_{k-1}\left(\lambda_{k-1}-1\right) \ldots\left(\lambda_{k-1}-(p-1)\right)}{(k-1)^{2}}\left(1-\frac{1}{\lambda_{k}}\right)^{\lambda_{k-1}}\right\} \\
& \leqq 2^{p}\left\{\sum_{n=1}^{k-2} \frac{\lambda_{k-1}\left(\lambda_{k-1}-1\right) \ldots\left(\lambda_{k-1}-(p-1)\right)}{(k-1)^{3}}+\frac{\lambda_{k-1}\left(\lambda_{k-1}-1\right) \ldots\left(\lambda_{k-1}-(p-1)\right)}{(k-1)^{2}}\right\} \\
& \leqq 2^{p}\left\{\frac{k-2}{(k-1)^{3}}+\frac{1}{(k-1)^{2}}\right\} \lambda_{k-1}\left(\lambda_{k-1}-1\right) \ldots\left(\lambda_{k-1}-(p-1)\right) .
\end{aligned}
$$

Hence

$$
|A| \leqq \frac{2^{p}(2 k-3)}{(k-1)^{3}} \lambda_{k-1}\left(\lambda_{k-1}-1\right) \ldots\left(\lambda_{k-1}-(p-1)\right)
$$

Inequality (8) implies that $\left(\lambda_{k-1} / \lambda_{k}\right) \rightarrow 0$ as $k \rightarrow \infty$. From this, (13) and (15) it follows that $(A / B) \rightarrow 0$ as $k \rightarrow \infty$. Therefore,

$$
|A| \leqq \frac{1}{4}|B|
$$

for all large $k$.

The function $y=x^{p} a^{x}$ for $x>0$, where $p>0$ and $0<a<1$, is increasing for $0<x<x_{0}$ and decreasing for $x>x_{0}$ where $x_{0}=-(p / \log \alpha)$. Since

$$
-\frac{p}{\log \left(1-\frac{1}{\lambda_{k}}\right)}=p \lambda_{k}+O(1) \quad \text { as } \quad k \rightarrow \infty \quad \text { and } \quad \lambda_{k+1} \geqq 2 p \lambda_{k}
$$

for large $k$ (because of $(8)$ ), the largest value of

$$
\lambda_{n}^{p}\left(1-\frac{1}{\lambda_{k}}\right)^{\lambda_{n}} \text { for } n \geqq k+1
$$

occurs when $n=k+1$. Hence, for all large $k$,(14) yields

$$
\begin{aligned}
|C| & \leqq 2^{p} \sum_{n=k+1}^{\infty} \frac{\lambda_{n}\left(\lambda_{n}-1\right) \ldots\left(\lambda_{n}-(p-1)\right)}{n^{2}}\left(1-\frac{1}{\lambda_{k}}\right)^{\lambda_{n}} \\
& \leqq 2^{p} \sum_{n=k+1}^{\infty} \frac{\lambda_{n}^{p}}{n^{2}}\left(1-\frac{1}{\lambda_{k}}\right)^{\lambda_{n}}
\end{aligned}
$$




$$
\begin{aligned}
& \leqq 2^{p} \lambda_{k+1}\left(1-\frac{1}{\lambda_{k}}\right)^{\lambda_{k+1}} \sum_{n=k+1}^{\infty} \frac{1}{n^{2}} \\
& \leqq 2^{p} \lambda_{+1}\left(1-\frac{1}{\lambda_{k}}\right)^{\lambda_{k+1}} \int_{k}^{\infty} \frac{1}{x^{2}} d x .
\end{aligned}
$$

Therefore, for all large $k$,

$$
|C| \leqq \frac{2^{p}}{k} \lambda_{k+1}^{p}\left(1-\frac{1}{\lambda_{k}}\right)^{\lambda_{k+1}}
$$

Let $\mu_{k}=\left(\lambda_{k+1} / \lambda_{k}\right)$. Then (8) implies that $\mu_{k} \geqq \lambda_{k} \geqq k$. Hence (17) shows that

$$
\begin{aligned}
\frac{|C|}{\frac{1}{k^{2}} \lambda_{k}^{p}} & \leqq 2^{p} k \mu_{k}^{p}\left(1-\frac{1}{\lambda_{k}}\right)^{\lambda_{k+1}} . \\
& \leqq 2^{p} \mu_{k}^{+1}\left[\left(1-\frac{1}{\lambda_{k}}\right)^{\lambda_{k}}\right]^{\mu_{k}}
\end{aligned}
$$

for all large $k$. Since

$$
\left(1-\frac{1}{\lambda_{k}}\right)^{\lambda_{k}} \rightarrow \frac{1}{e} \text { and } \lim _{n \rightarrow \infty} n^{q} a^{n}=0
$$

whenever $q>0$ and $0<a<1$, it follows that

$$
\frac{|C|}{\frac{1}{k^{2}} \lambda k} \rightarrow 0 \text { as } k \rightarrow \infty .
$$

Comparing this with (13), we conclude that $C / B \rightarrow 0$ as $k \rightarrow \infty$. Therefore for large $k$

$$
|C| \leqq \frac{1}{4}|B| .
$$

From (11), (16), (18) and (13) we find that for all large $k$,

$$
\left|f^{(p)}\left(r_{k} e^{i \theta}\right)\right| \geqq \frac{1}{2} \frac{a_{k}}{k^{2}\left(1-r_{k}\right)^{p}},
$$

where

$$
a_{k}=r_{k}\left(-1+2 r_{k}\right) \ldots\left(1-(p-1)+(p-1) r_{k}\right)\left(1-\frac{1}{\lambda_{k}}\right)^{\lambda_{k}-p}
$$


Since $a_{k} \rightarrow 1 / e$ as $k \rightarrow \infty, a_{k} \geqq \frac{1}{3}$ for large $k$. This, (9) and (19) yields the inequality (6).

The next result gives the sharp radial growth of the derivatives of a function subordinate to a univalent function. Again the exceptional sets are of measure zero.

Theorem 2. Suppose that the function $f$ is subordinate to some function which is analytic and univalent in $\Delta$. Then, for every positive integer $p$,

$$
\lim _{r \rightarrow 1^{-}}(1-r)^{p} f^{(p)}\left(r e^{i \theta}\right)=0
$$

for almost all $\theta$.

Also, let $\varepsilon$ be a positive nonincreasing function on $(0,1)$ such that $\lim _{r \rightarrow 1}-\varepsilon(r)=0$. Then there is a function $f$ which is subordinate to some function analytic and univalent in $\Delta$ for which (5) holds for every positive integer $p$.

Proof. If $F$ is analytic and univalent in $\Delta$, then $F$ belongs to the Hardy class $H^{q}$ for every $q<\frac{1}{2}[2$, p. 50]. This fact and Littlewood's inequality [2, p. 10] imply that if $f$ is subordinate to such an $F$ then $f \in H^{q}$ for $q<\frac{1}{2}$. Therefore $f$ has a nontangential limit as $z \rightarrow e^{i \theta}$ for almost all $\theta[2$, p. 17]. From our observations made earlier, this implies (20) for almost all $\theta$ and for $p=1,2, \ldots$.

To see that (5) holds for a suitable $f$, let $f$ be the function described by Theorem 1 and let $F(z)=M z$ where $M=\sup \{|f(z)|:|z|<1\}$. Since $f(0)=0, f$ is subordinate to $F$ and by Theorem $1,(5)$ holds for every positive integer $p$.

\section{Radial variation}

We next show that (3) and (4) are sharp. We present two examples of bounded analytic functions having such "maximal" growth. The reason for including both is their quite different character.

Theorem 3. There are positive constants $A_{p}$ and a function $f$ that is bounded and analytic in $\Delta$ such that

$$
(1-r)^{p} M\left(r, f^{(p)}\right) \geqq A_{p}
$$

for all $r, 0<r<1$ and for $p=1,2, \ldots$.

For each $\theta \in[0,2 \pi)$ there exists a function $f$ that is bounded and analytic in $\Delta$ such that

$$
\varliminf_{i \rightarrow 1^{-}}\left\{\frac{1}{\log \frac{1}{1-r}} \int_{0}^{r}\left|f^{(p)}\left(x e^{i \theta}\right)\right|^{\lambda} d x\right\}>0
$$

for $\lambda=1 / p$ and

$$
\varliminf_{r \rightarrow 1^{-}}\left\{(1-r)^{n \lambda-1} \int_{0}^{r}\left|f^{(p)}\left(x e^{i \theta}\right)\right|^{2} d x\right\}>0
$$

for $\lambda>1 / p$ and $p=1,2, \ldots$. 
Proof. For $|z|<1$, let $f(z)=(1-z)^{i}=\exp (i \log (1-z))$ where the logarithm is the principal branch. Since

$$
|\arg (1-z)|<\frac{\pi}{2} \text { for }|z|<1, e^{-\pi / 2}<|f(z)|<e^{\pi / 2} \text { for }|z|<1
$$

Hence $f$ is a bounded, analytic function and $|f(z)| \geqq A$ for $|z|<1$ where $A>0$. Now for $p=1,2, \ldots$ we have

$$
f^{(p)}(z)=a_{p} \frac{f(z)}{(1-z)^{p}}
$$

where $a_{p} \neq 0$, and thus

$$
\left|f^{(p)}(z)\right| \geqq A_{p} \frac{1}{|1-z|^{p}}
$$

where $A_{p}=\left|a_{p}\right| A>0$. Therefore,

$$
M\left(r, f^{(p)}\right) \geqq \frac{A_{p}}{(1-r)^{p}},
$$

and this proves (21).

From (24) we obtain

$$
\int_{0}^{r}\left|f^{(p)}(x)\right|^{\lambda} d x \geqq A_{p}^{\lambda} \int_{0}^{1} \frac{1}{(1-x)^{p \lambda}} d x .
$$

This implies (22) and (23) for $\theta=0$. To see that (22) and (23) hold for any $\theta$, it suffices to replace $f$ by $g$ where $g(z)=f\left(e^{-i \theta} z\right)$ since $\left|g^{(p)}\left(x e^{i \theta}\right)\right|=\left|f^{(p)}(x)\right|$.

When $p=1$ and $\lambda=1$, the precise value of $\int_{0}^{r}\left|f^{(p)}(x)\right|^{\gamma} d x$ for the function $f(z)=(1-z)^{i}$ can be obtained in the following way. As $x$ increases over the interval $[0, r], i \log (1-x)$ varies on the negative imaginary axis in a monotonic way from 0 to $i \log (1-r)$. Thus $f(x)$ varies along the unit circle clockwise starting at 1 and with an angular change equal to $\log (1-r)$. Hence the length of the curve $w=f(x), 0 \leqq x \leqq r$, equals $\log (1 / 1-r)$, that is

$$
\int_{0}^{r}\left|f^{\prime}(x)\right| d x=\log \frac{1}{1-r} .
$$

Our second example is an infinite Blashke product which satisfies (22) and (23) for $p=1$ and $\lambda \geqq 1$. For $n=1,2, \ldots$ let $a_{n}=1-\left(1 / 2^{n}\right)$ and let

$$
f(z)=\prod_{n=1}^{\infty} \frac{a_{n}-z}{1-a_{n} z}
$$

for $|z|=1$. Since $\sum_{n=1}^{\infty}\left(1-\left|a_{n}\right|\right)<+\infty, f$ is analytic in $\Delta$ and $|f(z)|<1$ for $|z|<1$. Let 


$$
r_{n}=\frac{1}{2}\left(a_{n}+a_{n+1}\right)=1-\frac{3}{2} \frac{1}{2^{n+1}} \text { for } n=1,2, \ldots
$$

Let $k$ be any positive integer. As $x$ varies on the interval $\left[a_{k}, a_{k+1}\right], f(x)$ varies from 0 to 0 and passes through $f\left(r_{k}\right)$. Hence the length of the curve $w=f(x), a_{k} \leqq x \leqq a_{k+1}$, is at least $2\left|f\left(r_{k}\right)\right|$. This is the same as

$$
\int_{a_{k}}^{a_{k}+1}\left|f^{\prime}(x)\right| d x \geqq 2\left|f\left(r_{k}\right)\right|
$$

Next we show that $\left|f\left(r_{k}\right)\right| \geqq A$ for $k=1,2, \ldots$ where

$$
A=\left[\prod_{n=0}^{\infty} \frac{1-\frac{3}{2}\left(\frac{1}{2}\right)^{n}}{1+\frac{3}{2}\left(\frac{1}{2}\right)^{n}}\right]^{2}
$$

We note that $A>0$. If $k<n$ then $r_{k}<a_{n}$ and it is easy to show that

$$
\left|\frac{a_{n}-r_{k}}{1-a_{n} r_{k}}\right| \geqq \frac{1-\frac{3}{2}\left(\frac{1}{2}\right)^{n-k-1}}{1+\frac{3}{2}\left(\frac{1}{2}\right)^{n-k-1}} .
$$

If $k \geqq n$ then $r_{k}>a_{n}$ and it follows that

$$
\left|\frac{a_{n}-r_{k}}{1-a_{n} r_{k}}\right| \geqq \frac{1-\frac{3}{2}\left(\frac{1}{2}\right)^{k-n+1}}{1+\frac{3}{2}\left(\frac{1}{2}\right)^{k-n+1}}
$$

These inequalities imply that

$$
\left|f\left(r_{k}\right)\right|=\prod_{n=1}^{k}\left|\frac{a_{n}-r_{k}}{1-a_{n} r_{k}}\right| \prod_{n=k+1}^{\infty}\left|\frac{a_{n}-r_{k}}{1-a_{n} r_{k}}\right| \geqq A .
$$

Since $\left|f\left(r_{k}\right)\right| \geqq A,(26)$ implies that

$$
\begin{aligned}
\int_{0}^{r_{n}}\left|f^{\prime}(x)\right| d x & \geqq \int_{a_{1}}^{a_{n}}\left|f^{\prime}(x)\right| d x=\sum_{k=1}^{n-1} \int_{a_{k}}^{a_{k}+1}\left|f^{\prime}(x)\right| d x \geqq \sum_{k=1}^{n-1} 2\left|f\left(r_{k}\right)\right| \\
& \geqq 2 A(n-1)=2 A\left[\frac{1}{\log 2}\left\{\log \frac{3}{2}+\log \frac{1}{1-r_{n}}\right\}-2\right] .
\end{aligned}
$$

Thus

$$
\int_{0}^{r_{n}}\left|f^{\prime}(x)\right| d x \geqq B \log \frac{1}{1-r_{n}}+C
$$

for constants $B$ and $C$ where $B>0$. 
Suppose that $\frac{5}{8} \leqq r<1$. Then $r_{n} \leqq r \leqq r_{n+1}$ for some $n$. Hence $(1-r) /\left(1-r_{n}\right) \geqq \frac{1}{2}$, and thus (27) implies that

$$
\int_{0}^{r}\left|f^{\prime}(x)\right| d x \geqq \int_{0}^{r_{n}}\left|f^{\prime}(x)\right| d x \geqq B \log \frac{1}{2}+B \log \frac{1}{1-r}+C
$$

Therefore (22) holds with $\lambda=p=1$.

Now suppose that $\gamma>1$. Hölder's inequality implies that

$$
\int_{a_{n}}^{a_{n+1}}\left|f^{\prime}(x)\right| d x \leqq\left(\int_{a_{n}}^{a_{n+1}}\left|f^{\prime}(x)\right|^{2} d x\right)^{1 / \lambda}\left(\int_{a_{n}}^{a_{n+1}} d x\right)^{1-(1 / \lambda)},
$$

and hence

$$
\begin{aligned}
\int_{a_{n}}^{a_{n+1}}\left|f^{\prime}(x)\right|^{\lambda} d x & \geqq\left(\int_{a_{n}}^{a_{n+1}}\left|f^{\prime}(x)\right| d x\right)^{\lambda} 2^{(n+1)(\lambda-1)} \geqq(2 A)^{\lambda} 2^{(n+1)(\lambda-1)} \\
& =(2 A)^{\lambda}\left(\frac{3}{2} \frac{1}{1-r_{n}}\right)^{\lambda-1} .
\end{aligned}
$$

Since $\int_{0}^{r_{n}+1}\left|f^{\prime}(x)\right|^{2} d x \geqq \int_{a_{n}}^{a_{n+1}}\left|f^{\prime}(x)\right|^{\lambda} d x$ this shows that

$$
\int_{0}^{r_{n+1}}\left|f^{\prime}(x)\right|^{\lambda} d x \geqq \frac{A_{\lambda}}{\left(1-r_{n}\right)^{\lambda-1}}
$$

where $A_{\lambda}>0$. Assume that $\frac{13}{16} \leqq r<1$. Then $r_{n+1} \leqq r \leqq r_{n+2}$ for some $n$ and thus $(1-r) /$ $\left(1-r_{n}\right) \geqq \frac{1}{4}$. Therefore, (28) implies that

$$
\int_{0}^{r}\left|f^{\prime}(x)\right|^{\lambda} d x \geqq \int_{0}^{r_{n}+1}\left|f^{\prime}(x)\right|^{\lambda} d x \geqq \frac{A_{\lambda}}{4^{\lambda-1}} \frac{1}{(1-r)^{\lambda-1}}
$$

This yields (23) when $p=1$.

We thank W. Rudin for the suggestion of looking at the function given in (25).

It is also possible to use infinite Blaschke products with appropriately chosen uniformly separated zeros to obtain for each $p$ a bounded analytic function $f$ satisfying $\lim _{r \rightarrow 1^{-}}(1-r)^{p} M\left(r, f^{(p)}\right)>0$. The argument is similar to that given for the example (25) and depends on the fact that for such a function $\left(1-|z|^{2}\right)^{p}\left|f^{(p)}(z)\right| \leqq B_{p}\left(1-|f(z)|^{2}\right)$ where $B_{p}>0[6$, p. 9]. These examples do not prove (21). They can be used to prove (22) and (23) for $p \geqq 2$ and $\lambda \geqq 1$. The argument uses the previous inequality and the inequality

$$
\int_{0}^{1}\left|f^{(p+1)}(x)\right| d x \geqq r\left|f^{(p)}(r)\right|-\int_{0}^{1}\left|f^{(p)}(x)\right| d x .
$$




\section{REFERENCES}

1. J. G. Clunie and T. H. MacGregor, Radial growth of the derivative of univalent functions, Comment. Math. Helv. 59 (1984), 362-375.

2. P. L. Duren, Theory of $H^{p}$ spaces (Academic Press, New York, 1970).

3. G. M. Goluzin, Geometric theory of functions of a complex variable (Amer. Math. Soc., Providence, 1969).

4. T. H. MacGregor, Growth of the derivatives of univalent and bounded functions, Ann. Univ. Mariae Curie Sklodowska, Sect. A 36/37 (1982-83), 101-113.

5. N. G. Makarov, On the distortion of boundary sets under conformal mappings, Proc. London Math. Soc. 51 (1985), 369-384.

6. St. RuscheweYh, Uber einige klassen im einheitskreis holomorpher funktionen (Math. Stat. Sektion Forschungszentrum Graz, Berich Nr. 7. 1974).

7. A. Zygmund, On certain integrals, Trans. Amer. Math. Soc. 55 (1944), 170-204.

Department of Mathematical Sciences

University of Delaware

Newark, Delaware 19716

U.S.A.
Department of Mathematics and Statistics SUNY at Albany 1400 Washington Avenue Albany, New York 12222 U.S.A. 